\title{
Effects of Abscisic Acid and Nitric Oxide on Chilling Resistance and Activation of the Antioxidant System in Walnut Shoots In Vitro
}

\author{
Ningguang Dong and Jianxun Qi \\ Key Laboratory of Urban Agriculture (North China), Ministry of Agriculture, Beijing Engineering \\ Research Center for Deciduous Fruit Trees, Institute of Forestry and Pomology, Beijing Academy of \\ Agriculture and Forestry Science, Beijing 100093, People's Republic of China
}

Yuanfa Li

Lab of Tree Breeding and Cultivation, State Forestry Administration, Research Institute of Forestry, Chinese Academy of Forestry, Beijing 100091, People's Republic of China

Yonghao Chen and Yanbin Hao'

Key Laboratory of Urban Agriculture (North China), Ministry of Agriculture, Beijing Engineering Research Center for Deciduous Fruit Trees, Institute of Forestry and Pomology, Beijing Academy of Agriculture and Forestry Science, Beijing 100093, People's Republic of China

\begin{abstract}
AdDitional INDEX words. antioxidant defense, chlorophyll fluorescence, Juglans regia, reactive oxygen species, sodium nitroprusside

Abstract. The roles of abscisic acid (ABA) and nitric oxide (NO) and the relationship between NO and ABA on chilling resistance and activation of antioxidant activities in walnut (Juglans regia) shoots in vitro under chilling stress were investigated. Walnut shoots were treated with ABA, the NO donor sodium nitroprusside (SNP), ABA in combination with the NO scavenger 2-phenyl-4,4,5,5-tetramethyl-imidazoline-1-oxyl-3-oxide (PTIO), PTIO, SNP in combination with the ABA biosynthesis inhibitor fluridone (Flu), and Flu. Their effects on chilling tolerance, reactive oxygen species (ROS) levels, and the antioxidant defense system were analyzed. The results showed that ABA treatment markedly alleviated the decreases in the maximal photochemical efficiency and survival and the increases in electrolyte leakage and lipid peroxidation induced by chilling stress, suggesting that application of ABA could improve the chilling tolerance. Further analyses showed that $A B A$ enhanced antioxidant defense and slowed down the accumulation of ROS caused by chilling. Similar results were observed when exogenous SNP was applied. ABA in combination with PTIO or PTIO alone differentially abolished these protective effects of ABA. However, treatment with NO in combination with Flu or Flu alone did not affect the SNP-induced protective effect against CI or the activation of antioxidant activities under conditions of chilling stress. In addition, ABA treatment increased the NO content under chilling conditions, which was suppressed by the ABA biosynthesis inhibitor Flu or NO scavenger PTIO. Conversely, SNP application induced the same ABA rise observed in control plants in response to chilling. Taken together, these results suggested that $\mathrm{ABA}$ may confer chilling tolerance in walnut shoots in vitro by enhancing the antioxidant defense system, which is partially mediated by NO, preventing the overproduction of ROS to alleviate the oxidative injury induced by chilling.
\end{abstract}

Among the various types of environmental stress, chilling stress is one of the most important factors limiting the productivity and distribution of plants. Chilling temperatures, defined as low but not freezing temperatures $\left(0\right.$ to $\left.15^{\circ} \mathrm{C}\right)$, are common in nature and can cause a complex array of cellular dysfunctions, including loss of vigor, wilting, chlorosis, sterility, and even death (Dong et al., 2014; Theocharis et al., 2012). Oxidative stress may be a significant factor involved in chilling-induced injury (Zhou and Guo, 2009). Several studies have demonstrated that exposure to low temperatures induces the accumulation of ROS, such as $\mathrm{H}_{2} \mathrm{O}_{2}$, singlet oxygen,

Received for publication 30 June 2017. Accepted for publication 21 July 2017. We sincerely thank Dong Pei (Lab of Tree Breeding and Cultivation, State Forestry Administration, Research Institute of Forestry, Chinese Academy of Forestry) for her kind help with experiment design and the equipment. This work was supported by the National Natural Science Foundation of China (Grant No. 31401817) and the Commonweal Special Foundation of State Forestry Administration of China (No. 201304712).

${ }^{1}$ Corresponding author. E-mail: jinhetaojht@263.net. superoxide radicals $\left(\mathrm{O}_{2}^{-}\right)$, and hydroxyl radicals $\left(\mathrm{OH}^{-}\right)$, which could cause oxidative stress at the cellular level (Hu et al., 2017; Liu et al., 2011; Theocharis et al., 2012). To scavenge ROS and combat oxidant damage, plants have evolved an antioxidant defense system consisting of several antioxidant enzymes; i.e., superoxide dismutase (SOD), ascorbate peroxidase (APX), glutathione reductase (GR), and catalase (CAT), and some low molecular weight nonenzymatic antioxidants, such as ascorbic acid (AsA) and reduced glutathione (GSH) (Liu et al., 2010). Membranes are the primary sites where responses to chilling-induced oxidative stress occur. Electrolyte leakage and malondialdehyde (MDA) content are often used to assess stress-induced damage in membranes (Karimi and Ershadi, 2015; Zhang et al., 2012). Chloroplasts are among the most susceptible cellular organelles, and chilling leads to a decrease in the photosystem II maximal photochemical efficiency, which is generally expressed as the ratio of variable to maximal fluorescence ratio $\left(F_{\mathrm{v}} / F_{\mathrm{m}}\right)$ (Wang et al., 2016). 
Abscisic acid acts as a stress signal and plays critical roles in the tolerance of plants to many stress factors (Venzhik et al., 2016). ABA induces a myriad of cellular responses in plants via complex signal transduction cascades, leading to tolerance to stress conditions (Zhang et al., 2007). In plant tissues, the increase in endogenous ABA content after exposure to cold temperatures is assumed to be associated with the subsequent increase in the chilling tolerance of plants (Karimi and Ershadi, 2015). In addition, application of exogenous ABA increased the chilling tolerance in various herbaceous plants (Guo et al., 2012; Meng et al., 2008; Zhang et al., 2007). Although ABA is typically considered a key regulator of many abiotic stress responses, the mechanism underlying plant responses to abiotic stress are not exclusively controlled by ABA signaling. Under conditions of environmental stress, ABA interacts with other endogenous signaling substances (Asgher et al., 2017; Freschi, 2013). Among these ABA-interacting substances, the gaseous free radical NO has recently attracted interest in the research community given its involvement in a number of signaling cascades controlling plant responses to abiotic stress (Fancy et al., 2017; Farnese et al., 2016).

Nitric oxide, which was first identified as a unique diffusible molecular messenger in animals, plays important roles in various plant physiological processes, including plant growth, development, and the response to abiotic stress (Fancy et al., 2017; Liu et al., 2010; Sarath et al., 2007). Because of its biological action, NO has been considered either a stressinducing or protective agent. For example, under conditions of environmental stress, application of the NO donor SNP in plants resulted in increased tolerance to environmental stresses, thereby alleviating injury and promoting plant growth (Asgher et al., 2017). Both NO and ABA, important "stress-related" molecules, interact during certain signaling cascades triggered by environmental challenges, such as water limitation and salt stress, ultimately leading to induction of plant adaptive responses, such as stomatal closure and antioxidant defenses (Freschi, 2013; Manai et al., 2014; Neill et al., 2008). However, few studies have investigated the interaction between NO and ABA during plant responses to cold temperatures. More evidence is required regarding the existence of $\mathrm{NO}-\mathrm{ABA}$ interactions in chilling resistance and antioxidant defense in plants.

Walnut, one of the most economically important crops for both its timber and edible nuts, is susceptible to CI by spring frost. Therefore, it is important to explore the mechanism underlying acclimation to cold temperatures in walnut. In the present study, walnut shoots were treated in vitro with ABA, SNP, the NO scavenger PTIO, and the ABA biosynthesis inhibitor Flu. First, the effects of ABA and NO on chilling resistance, ROS levels, and the antioxidant defense system were investigated in walnut shoots in vitro under conditions of chilling stress. The existence of NO-ABA interaction in chilling resistance and antioxidant defense in walnut shoots under conditions of chilling stress were also evaluated in vitro.

\section{Materials and Methods}

Plant material and treatments. The walnut cultivar Jinlong 2 was cultured aseptically on proliferation medium [full-strength Driver and Kuniyuki (DKW) medium supplemented with $4.4 \mu \mathrm{M}$ 6-benzyl-aminopurine and $4.9 \mathrm{~nm} 3$-indolebutyric acid] as described previously (Pei et al., 2007). The medium was autoclaved at $121{ }^{\circ} \mathrm{C}$ and $124 \mathrm{kPa}$ for $20 \mathrm{~min}$ before use. Subculturing was performed every $20 \mathrm{~d}$. Shoot proliferation was performed in $350-\mathrm{mL}$ cylindrical bottles. The cultures were incubated at $25 \pm 3{ }^{\circ} \mathrm{C}$ under a 16 -h photoperiod $\left(53 \mu \mathrm{mol} \cdot \mathrm{m}^{-2} \cdot \mathrm{s}^{-1}\right)$ provided by cool-white fluorescent tubes. As shown in Fig. 1, shoots, $4.5-5.0 \mathrm{~cm}$ in length, were excised and used for all investigations.

To investigate the effects of ABA or SNP on chilling tolerance in walnut shoots, walnut shoots, $4.5-5.0 \mathrm{~cm}$ in length, were excised and transferred into proliferation medium with ABA of different concentration $(0,100,200$, and $400 \mu \mathrm{M})$ or SNP of different concentration $(0,50,100$, and $200 \mu \mathrm{M})$. Subsequently, the treated shoots were cultured in 25 or $4{ }^{\circ} \mathrm{C}$ for $3 \mathrm{~d}$. PTIO was used as the NO scavenger and Flu as the ABA biosynthesis inhibitor. To elaborate the influence of exogenously applied PTIO on the NO content or exogenously applied Flu on the ABA content, walnut shoots, $4.5-5.0 \mathrm{~cm}$ in length, were treated with PTIO of different concentration $(0,100,200$, and $400 \mu \mathrm{M})$ or Flu of different concentration $(0,50,100$, and $200 \mu \mathrm{M})$ and then cultured in 25 or $4{ }^{\circ} \mathrm{C}$ for $3 \mathrm{~d}$.

To investigate the existence of $\mathrm{NO}-\mathrm{ABA}$ interaction in chilling resistance and antioxidant defense in walnut shoots under conditions of chilling stress, the walnut shoots, 4.5$5.0 \mathrm{~cm}$ in length, were excised and transferred into different proliferation medium containing the following: 1) sterilized water (control), 2) $200 \mu \mathrm{M} \mathrm{ABA,} \mathrm{3)} 100 \mu \mathrm{M} \mathrm{SNP,} \mathrm{4)} 200 \mu \mathrm{M}$ $\mathrm{ABA}+200 \mu \mathrm{M}$ PTIO, 5) $200 \mu \mathrm{M}$ PTIO, 6) $100 \mu \mathrm{M} \mathrm{SNP}+50 \mu \mathrm{M}$ Flu, or 7) $50 \mu \mathrm{M}$ Flu. Half of the plants in each treatment were cultured at $25^{\circ} \mathrm{C}$, the other half of plants were exposed to $4{ }^{\circ} \mathrm{C}$. After $3 \mathrm{~d}$ of culture, the third leaves were sampled for

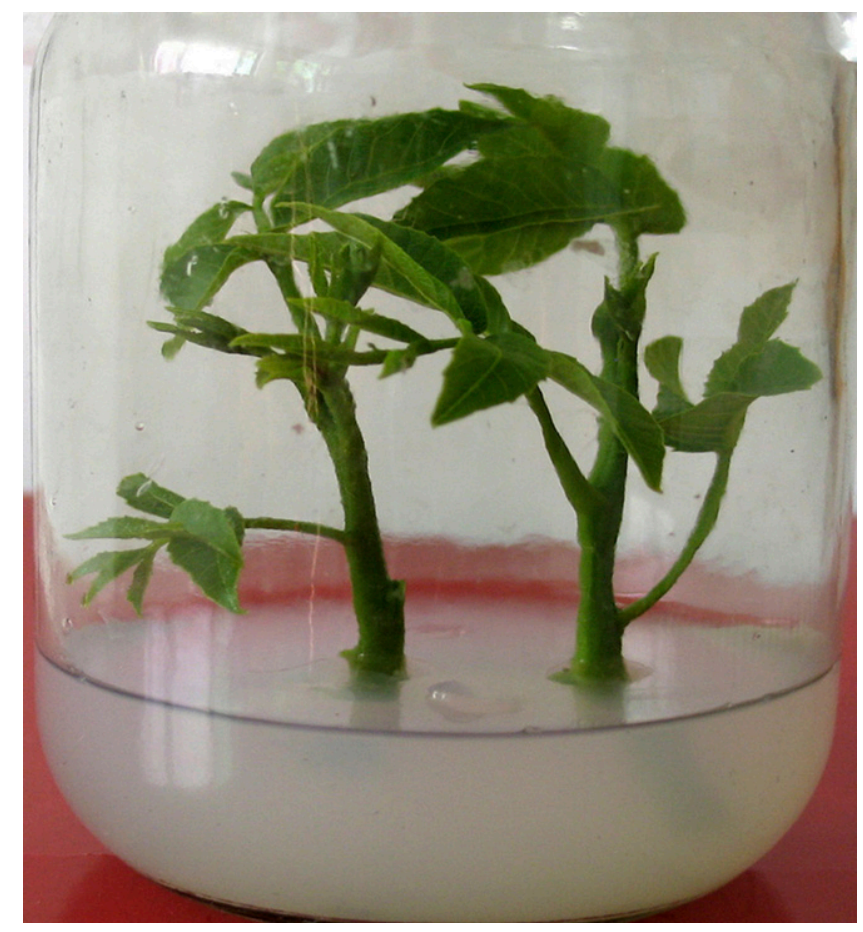

Fig. 1. In vitro walnut shoots of $4.5-5.0 \mathrm{~cm}$ height used for the investigation of effects of abscisic acid (ABA), sodium nitroprusside, 2-phenyl4,4,5,5-tetramethyl-imidazoline-1-oxyl-3-oxide, and fluridone on the electrolyte leakage, malondialdehyde content, maximal fluorescence ratio, percentage survival, nitric oxide contents, and $\mathrm{ABA}$ contents in the leaves of walnut shoots in vitro after $3 \mathrm{~d}$ of cultivation at 25 or $4{ }^{\circ} \mathrm{C}$. 
measurement. Fresh leaves were used for electrolyte leakage and maximal photochemical efficiency measurements, and the others were immediately frozen in liquid $\mathrm{N}_{2}$ for analysis of other physiological parameters. In addition, the percentage of plant survival for each treatment was recorded.

Measurement of electrolyte LeAKage. Electrolyte leakage was determined as described by Soloklui et al. (2012) with slight modifications. Fresh leaves $(0.5 \mathrm{~g})$ were cut into $1-\mathrm{cm}^{2}$ pieces and placed in test tubes containing $40 \mathrm{~mL}$ distilled deionized water for $24 \mathrm{~h}$ on a shaker at $120 \mathrm{rpm}$. The electrical conductivity $\left(\mathrm{EC}_{1}\right)$ of the solutions was measured using a conductivity meter (Cond 720; WTW, Weilheim, Germany). The samples were boiled at $100{ }^{\circ} \mathrm{C}$ for $20 \mathrm{~min}$ to release all electrolytes and then cooled to room temperature, after which the final $\mathrm{EC}\left(\mathrm{EC}_{2}\right)$ was measured. Electrolyte leakage was calculated using the following formula: $\left(\mathrm{EC}_{1} / \mathrm{EC}_{2}\right) \times 100$.

Measurement of MDA. Malondialdehyde content was measured according to a method described previously (Liu et al., 2011). Freeze-dried and powdered leaf mass $(0.1 \mathrm{~g})$ was homogenized in 5\% trichloroacetic acid (TCA) and then was centrifuged at $1000 g_{\mathrm{n}}$ for $10 \mathrm{~min}$. The supernatant was mixed with the equal volume of 5\% TCA containing $0.67 \%$ thiobarbituric acid (TBA). The mixture was incubated in a boiling water bath for $30 \mathrm{~min}$ and quickly cooled to room temperature. Optical density (OD) at 450, 532, and $600 \mathrm{~nm}$ was measured after centrifugation at $1000 g_{n}$ for $10 \mathrm{~min}$ again. MDA content was calculated using the formula: $\mathrm{MDA}(\mu \mathrm{M})=\left[6.45\left(\mathrm{OD}_{532}-\mathrm{OD}_{600}\right)-\right.$ $\left.0.56 \mathrm{OD}_{450}\right]$.

Analysis of THE MAXimal PHOTOCHEMICAL EFFICIENCY OF РнOTOSYSTEM II. A fluorometer (FMS-1; Hansatech Instruments, Norfolk, UK) was used to measure modulated chlorophyll fluorescence. The leaves were excised and immediately mounted to the fluorometer. For dark adaptation, leaves were covered for $30 \mathrm{~min}$. The initial fluorescence $\left(F_{0}\right)$ was measured at a photosynthetic photon flux (PPF) density of $<0.05 \mu \mathrm{mol} \cdot \mathrm{m}^{-2} \cdot \mathrm{s}^{-1}$, followed by a saturating pulse $\left(3000 \mu \mathrm{mol} \cdot \mathrm{m}^{-2} \cdot \mathrm{s}^{-1}\right)$ to determine the maximal fluorescence $\left(F_{\mathrm{m}}\right)$. The maximal photochemical efficiency of photosystem II was estimated using the following formula: $F_{\mathrm{v}} / F_{\mathrm{m}}=\left(F_{\mathrm{m}}-F_{0}\right) / F_{\mathrm{m}}$.

Recording of Survival. After $3 \mathrm{~d}$ of treatment, the shoots subjected to $4{ }^{\circ} \mathrm{C}$ were moved to $25{ }^{\circ} \mathrm{C}$, and the other shoots were still kept at $25{ }^{\circ} \mathrm{C}$. The survival of each shoot was monitored daily for $20 \mathrm{~d}$. The number of shoots used was not less than 30 for each treatment.

Determination of ABA content. ABA content was determined using the high performance liquid chromatographymass spectrometry (HPLC-MS) method described by Gou et al. (2010). A 100-mg aliquot of freeze-dried and powdered leaf mass was extracted in $80 \%$ methanol with internal standards of $\left[{ }^{2} \mathrm{H}_{2}\right]-\mathrm{ABA}$ and reduced to aqueous phase. The aqueous phase was extracted with ethyl acetate at $\mathrm{pH} 3$, then with potassium phosphate buffer at $\mathrm{pH} 8.5$, again into ethyl acetate at $\mathrm{pH} 3$, and further purified on $\mathrm{C}_{18}$ Sep-Pak and Qasis MCX SPE columns (Waters, Milford, MA). The eluant was dried and redissolved with HPLC initial solution, filtered through a $0.22-\mu \mathrm{m}$ filter, and analyzed with a liquid chromatography-mass spectrometry system (LCQ Deca AMX, HPLCESI-MS; Thermo Fisher Scientific, Waltham, MA). Tandem mass spectrometry data were then analyzed using software Xcalibur 2.1 (Thermo Fisher Scientific) and quantified by reference to the internal standards using $\mathrm{M}^{+}$ratios in equations for isotope dilution analysis.
Determination of NO content. NO content was determined using the method described by Ziogas et al. (2013) with slight modifications. Freeze-dried and powdered leaf mass $(0.1 \mathrm{~g})$ was homogenized in cool acetic acid buffer (containing $4 \%$ zinc diacetate) at $\mathrm{pH} 3.6$. The homogenates were centrifuged at $10,000 \mathrm{~g}_{\mathrm{n}}$ for $10 \mathrm{~min}$ at $4{ }^{\circ} \mathrm{C}$. The supernatant was collected. The pellet was washed by $1 \mathrm{~mL}$ of extraction buffer and centrifuged again as before. The two supernatants were combined and clarified with $0.1 \mathrm{~g}$ charcoal. After vortex and filtration, the filtrate was leached and collected. The mixture of $1 \mathrm{~mL}$ of filtrate and $1 \mathrm{~mL}$ of the Greiss reagent was incubated at room temperature for $30 \mathrm{~min}$. Absorbance was measured at $540 \mathrm{~nm}$. NO content was calculated by comparison with a standard curve using sodium nitrite.

Determination of ROS PRODUCTION. The $\mathrm{H}_{2} \mathrm{O}_{2}$ content was determined according to a method described previously (Zhou et al., 2006). Freeze-dried and powdered leaf mass $(0.1 \mathrm{~g})$ was homogenized in 5\% TCA and $0.1 \mathrm{~g}$ activated charcoal. Following centrifugation $\left(10,000 \mathrm{~g}_{\mathrm{n}}, 20 \mathrm{~min}, 4{ }^{\circ} \mathrm{C}\right)$, the supernatant was adjusted to $\mathrm{pH} 8.4$ with $17 \mathrm{M}$ ammonia solution and then filtered. The filtrate was divided into aliquots of $1 \mathrm{~mL}$. To one of these, the blank was added $8 \mu \mathrm{g}$ of CAT and then kept at room temperatures for $10 \mathrm{~min}$. To both aliquots with and without CAT, $1 \mathrm{~mL}$ of colorimetric reagent was added. The reaction solution was incubated for $10 \mathrm{~min}$ at $30{ }^{\circ} \mathrm{C}$. Absorbance at $505 \mathrm{~nm}$ was determined spectrophotometrically (model ultraviolet-2010; Hitachi, Tokyo, Japan).

The $\mathrm{O}_{2}{ }^{-}$production rate was measured using the method of Zhao et al. (2008) with slight modifications. Freeze-dried and powdered leaf mass $(0.2 \mathrm{~g})$ was homogenized in $4 \mathrm{~mL}$ of $65 \mathrm{~mm}$ potassium phosphate buffer $(\mathrm{pH} 7.8)$ and centrifuged at $5000 \mathrm{~g}_{\mathrm{n}}$ for $10 \mathrm{~min}$. The incubation mixture contained $0.9 \mathrm{~mL}$ of $65-\mathrm{mm}$ potassium phosphate buffer ( $\mathrm{pH} 7.8), 0.1 \mathrm{~mL}$ of $10-\mathrm{mm}$ hydroxylammonium chloride and $1 \mathrm{~mL}$ of the supernatant. After incubation at $25{ }^{\circ} \mathrm{C}$ for $20 \mathrm{~min}, 17-\mathrm{mm}$ sulphanilic acid and 7-mm a-naphthyl amine were added to the incubation mixture. After reaction at $25{ }^{\circ} \mathrm{C}$ for a further $20 \mathrm{~min}$, the absorbance was read at $530 \mathrm{~nm}$. A standard curve with sodium nitrite was used to calculate the production rate of $\mathrm{O}_{2}^{-}$.

The content of $\mathrm{OH}^{-}$was determined using the method described by Liu et al. (2011) with slight modifications. Freeze-dried and powdered leaf mass $(0.1 \mathrm{~g})$ was homogenized with $1.2 \mathrm{~mL}$ of 50-mm sodium phosphate buffer $(\mathrm{pH} 7.0)$ and centrifuged at $10,000 \mathrm{~g}_{\mathrm{n}}$ for $10 \mathrm{~min}$. The supernatant was mixed with $0.5 \mathrm{~mL}$ of 50-mm sodium phosphate buffer $(\mathrm{pH} \mathrm{7.0)}$ and $1 \mathrm{~mL}$ of $25-\mathrm{mm}$ sodium phosphate buffer ( $\mathrm{pH} 7.0)$ containing 2.5-mм 2-deoxyribose. The reaction was developed at $35^{\circ} \mathrm{C}$ in the dark for $1 \mathrm{~h}$. After adding $1 \mathrm{~mL}$ of $1 \%$ TBA in $0.05 \mathrm{M}$ sodium hydroxide and $1 \mathrm{~mL}$ of acetic acid, the mixture was boiled for $30 \mathrm{~min}$ and immediately cooled for $10 \mathrm{~min}$ in ice. The production of $\mathrm{OH}^{-}$was followed by measurement of absorbance at $532 \mathrm{~nm}$, and the results were given as absorbance units (absorbance $\times 1000$ ) per gram sample dry weight.

Antioxidant ENZYMe aCtivity ASSAY. Freeze-dried and powdered leaf mass $(0.1 \mathrm{~g})$ was homogenized in $5 \mathrm{~mL}$ of 50-mm chilled phosphate buffer ( $\mathrm{pH} 7.0$, containing 1-mm AsA and 1-mm ethylenediaminetetraacetic acid). The supernatants were recovered for determination of SOD, CAT, APX, and GR activities as described previously (Guo et al., 2006; Zhang et al., 2009; Zhou et al., 2005). One unit of CAT, APX, or GR was defined as the amount of enzyme required to catalyze the conversion of $1-\mu \mathrm{mol} \mathrm{H}_{2} \mathrm{O}_{2}$ (extinction coefficient $0.0394 \mathrm{~mm}^{-1} \cdot \mathrm{cm}^{-1}$ ), 
AsA (extinction coefficient $2.8 \mathrm{~mm}^{-1} \cdot \mathrm{cm}^{-1}$ ), or nicotinamide adenine dinucleotide phosphate (extinction coefficient $6.22 \mathrm{~mm}^{-1} \cdot \mathrm{cm}^{-1}$ ), respectively, within $1 \mathrm{~min}$. One unit of SOD activity was defined as the amount of enzyme required for inhibition of photochemical reduction of r-nitro blue tetrazolium chloride by $50 \%$. Protein content was determined using Coomassie Brilliant Blue G-250 according to the method of Bradford (1976).

Measurement of antioxidant Content. Freeze-dried and powdered leaf mass samples $(0.1 \mathrm{~g})$ were homogenized in $5-\mathrm{mL}$ TCA at $4{ }^{\circ} \mathrm{C}$. The homogenate was centrifuged at $13,000 g_{\mathrm{n}}$ for $15 \mathrm{~min}$. The supernatant was recovered and used for AsA and GSH determination according to methods described previously (Griffith, 1980; Law et al., 1983).

Statistical analysis. All treatments were repeated at least three times, and all samples were analyzed three times. At least 30 shoots were included for each treatment. The data were subjected to analysis of variance, and significant differences between means were determined using Duncan's multiplerange test. In all analyses, $P<0.05$ was taken to indicate statistical significance.

\section{Results}

ABA OR SNP TREATMENT ENHANCES THE TOLERANCE OF WALNUT SHOOTS TO CHILLING STRESS. A preliminary experiment with different concentrations of ABA or SNP was performed to determine the optimal concentration that ABA or SNP showed the most significant effect. As shown in Fig. 2, chilling treatment caused significant increases in electrolyte leakage
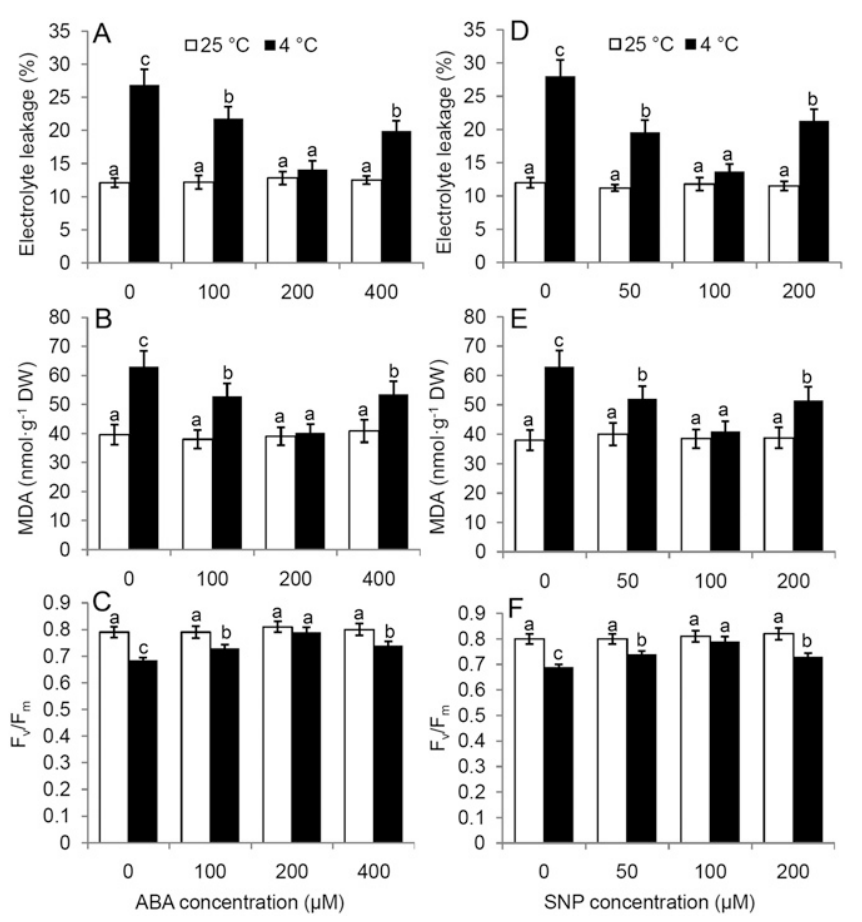

Fig. 2. Effects of different abscisic acid (ABA) concentrations on electrolyte leakage (A), malondialdehyde (MDA) content (B) and maximal fluorescence ratio $\left(F_{\mathrm{v}} / F_{\mathrm{m}}\right)(\mathbf{C})$ and different SNP concentrations on electrolyte leakage (D), MDA content $(\mathbf{E})$, and $F_{\mathrm{v}} / F_{\mathrm{m}}(\mathbf{F})$ in the leaves of walnut shoots in vitro after $3 \mathrm{~d}$ of cultivation at 25 or $4{ }^{\circ} \mathrm{C}$. Values represent the means of three replicates $\pm \mathrm{SD}$ as shown by the vertical error bars. Different letters above the bars indicate significant differences via Duncan's multiple-range test at $P<0.05$. and MDA content and a decrease in the $F_{\mathrm{v}} / F_{\mathrm{m}}$. Application of ABA or SNP with different concentrations had no significant effects on electrolyte leakage, MDA content, and $F_{\mathrm{v}} / F_{\mathrm{m}}$ at $25{ }^{\circ} \mathrm{C}$ (Fig. 1). However, under low temperature conditions, treatment with ABA or SNP markedly alleviated the decrease in the $F_{\mathrm{v}} / F_{\mathrm{m}}$ and the increases in electrolyte leakage and MDA content induced by chilling stress (Fig. 1). Among the different concentrations of ABA or SNP, $200 \mu \mathrm{M}$ ABA, or $100 \mu \mathrm{M}$ SNP showed the most significant alleviation effect on CI in the walnut shoots. Therefore, $200-\mu \mathrm{M}$ ABA or $100-\mu \mathrm{M}$ SNP was used in further experiments.

PTIO decreases NO Content and Flu decreases ABA CONTENT. As shown in Fig. 3A, the decrease of NO content was observed when varying concentrations of PTIO were applied under 25 or $4{ }^{\circ} \mathrm{C}$. Along with the increased concentration of PTIO, there was a strong tendency toward the decrease of NO content (Fig. 3A). However, with the concentration of PTIO increased from 200 to $400 \mu \mathrm{M}$, there was no obvious decrease in NO content under 25 or $4{ }^{\circ} \mathrm{C}$ (Fig. 3A). Therefore, 200- $\mu_{\mathrm{M}}$ PTIO was used in further experiments. Similarly, application of different concentrations of Flu decreased the ABA content under 25 or $4{ }^{\circ} \mathrm{C}$ (Fig. 3B). The amount of ABA was negatively correlated with the Flu concentration. Under low temperature, the $\mathrm{ABA}$ content dropped obviously when treated with $50 \mu_{\mathrm{M}} \mathrm{Flu}$, compared with the control treated without Flu (Fig. 3B). When the concentration of Flu increased to 100 or $200 \mu \mathrm{M}$, the decrease in ABA content was not obvious under 25 or $4{ }^{\circ} \mathrm{C}$ (Fig. 3B). Thus, $50-\mu \mathrm{M}$ Flu was used in further experiments.

Effects of ABA, SNP, PTIO, and Flu on Chilling TOLERANCE, ABA CONTENTS, AND NO CONTENTS. To investigate the effects of ABA, SNP, PTIO, and Flu on the tolerance of walnut shoots to chilling stress, it was necessary to evaluate the phenotypical and physiological features. Figure 4 shows the effects of different treatments on phenotypes in leaves of walnut shoots as evidenced by typical visual injury symptoms of the corresponding treatment imposed.

As shown in Fig. 5A and B, the electrolyte leakage and MDA content increased by $133.3 \%$ and $65.8 \%$ under conditions of chilling stress at $4{ }^{\circ} \mathrm{C}$, respectively. Figure $5 \mathrm{C}$ shows that the $F_{\mathrm{v}} / F_{\mathrm{m}}$ ratio and percentage survival decreased by $12.5 \%$ and $32 \%$ under chilling stress at $4{ }^{\circ} \mathrm{C}$. Application of ABA or SNP significantly alleviated the increases of electrolyte leakage and MDA content and the suppression of $F_{\mathrm{v}} / F_{\mathrm{m}}$ and survival induced by chilling stress (Fig. 5A-D). Under low temperature, treatment with NO scavenger PTIO or ABA synthesis inhibitor Flu alone differentially abolished those protective effects of
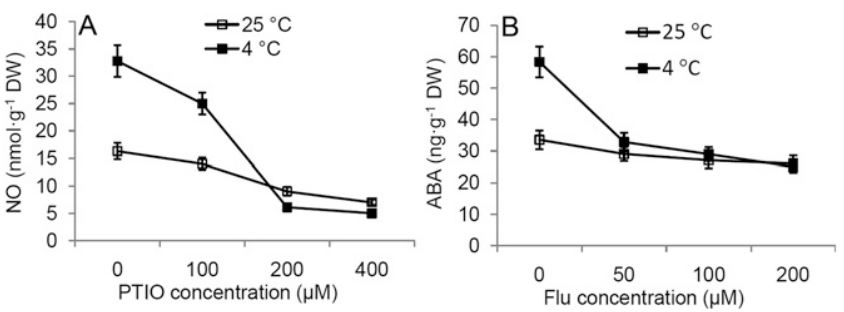

Fig. 3. Effects of different 2-phenyl-4,4,5,5-tetramethyl-imidazoline-1-oxyl-3oxide (PTIO) concentrations on nitric oxide (NO) content (A) and different fluridone (Flu) concentrations on abscisic acid content (B) in the leaves of walnut shoots in vitro after $3 \mathrm{~d}$ of cultivation at 25 or $4{ }^{\circ} \mathrm{C}$. Each value represents mean of three replicates \pm SD shown by vertical error bar. 


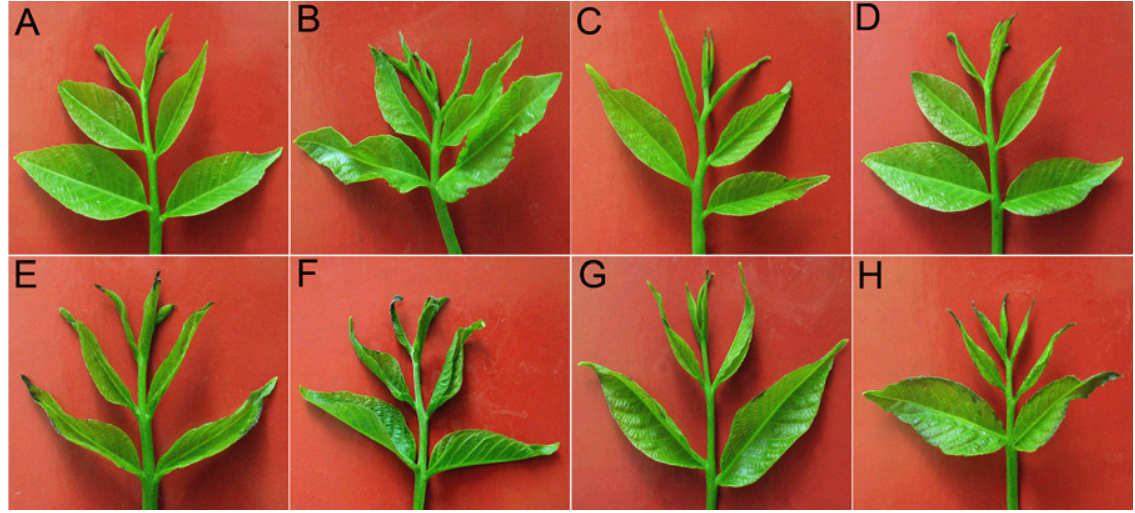

Fig. 4. Phenotypes in leaves of walnut shoots in vitro subjected to different treatments for $3 \mathrm{~d}$ : (A) control, (B) chilling, (C) chilling + abscisic acid (ABA), (D) chilling + sodium nitroprusside (SNP), (E) chilling + ABA + 2-phenyl-4,4,5,5-tetramethyl-imidazoline-1-oxyl-3-oxide (PTIO), (F) chilling + PTIO, (G) chilling + SNP + fluridone (Flu), and (H) chilling + Flu.
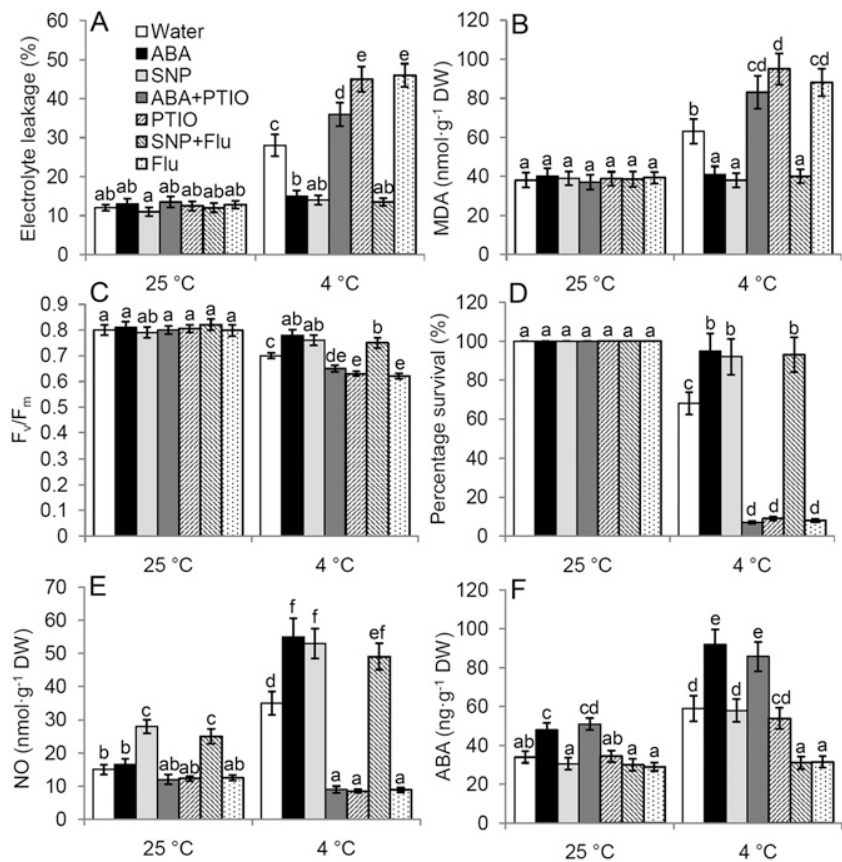

Fig. 5. Effects of abscisic acid (ABA), sodium nitroprusside (SNP), 2-phenyl4,4,5,5-tetramethyl-imidazoline-1-oxyl-3-oxide (PTIO), and fluridone (Flu) on the electrolyte leakage (A), malondialdehyde (MDA) content (B), maximal fluorescence ratio $\left(F_{\mathrm{v}} / F_{\mathrm{m}}\right)(\mathbf{C})$, percentage survival $(\mathbf{D})$, nitric oxide (NO) contents $(\mathbf{E})$, and ABA contents $(\mathbf{F})$ in the leaves of walnut shoots in vitro after $3 \mathrm{~d}$ of cultivation at 25 or $4{ }^{\circ} \mathrm{C}$. Values represent the means of three replicates $\pm \mathrm{SD}$ as shown by the vertical error bars. Different letters above the bars indicate significant differences via Duncan's multiple-range test at $P<0.05$.

ABA or SNP (Fig. 5A-D). Moreover, ABA in combination with PTIO also arrested the ABA- or SNP-mediated protective effects (Fig. 5A-D). That is, in the presence of $\mathrm{ABA}$ in combination with PTIO, PTIO, or Flu, electrolyte leakage and MDA content increased markedly and $F_{\mathrm{v}} / F_{\mathrm{m}}$ and survival decreased noticeably compared with those exposed to chilling stress alone. However, under low temperature, SNP in combination with Flu completely prevented the increased electrolyte leakage and MDA content and decreased $F_{\mathrm{v}} / F_{\mathrm{m}}$ and survival induced by chilling stress (Fig. 5A-D). In addition, walnut shoots in vitro showed no obvious differences in the previously mentioned physiological parameters among all of the treatments at $25^{\circ} \mathrm{C}$ (Fig. 5A-D).

Figure 5E shows that chilling stress induced a marked increase in endogenous NO production. Under low temperature, NO content increased noticeably in the presence of SNP or ABA compared with that of chilling stress alone, whereas PTIO or Flu completely inhibited the increase in NO content (Fig. $5 \mathrm{E})$. In addition, under low temperature, SNP in combination with Flu markedly increased the NO content compared with chilling stress alone, whereas $\mathrm{ABA}$ in combination with PTIO caused a significant decrease in NO content (Fig. 5E).

Figure $5 \mathrm{~F}$ shows that the endogenous $\mathrm{ABA}$ content in walnut shoots in vitro increased by $84.4 \%$ under chilling stress at $4{ }^{\circ} \mathrm{C}$. Under conditions of chilling stress, the application of $\mathrm{ABA}$ or $\mathrm{ABA}$ in combination with PTIO induced a marked increase in ABA content compared with chilling stress alone (Fig. 5F). However, under low temperature, SNP or PTIO treatment induced the same ABA rise observed in control plants in response to chilling (Fig. 5F). In addition, SNP in combination with Flu or Flu markedly inhibited the chilling stress-induced increase in $\mathrm{ABA}$ (Fig. 5F).

Effects of ABA, SNP, PTIO, and Flu on ROS levels. Exposure of walnut shoots to low temperature in vitro led to marked increases in $\mathrm{H}_{2} \mathrm{O}_{2}, \mathrm{O}_{2}^{-}$, and $\mathrm{OH}^{-}$contents. As shown in Fig. $6 \mathrm{~A}-\mathrm{C}, \mathrm{H}_{2} \mathrm{O}_{2}, \mathrm{O}_{2}^{-}$, and $\mathrm{OH}^{-}$contents increased by $175 \%$, $62.5 \%$, and $27.4 \%$, respectively, under chilling stress at $4{ }^{\circ} \mathrm{C}$. Exogenous ABA or SNP treatment significantly alleviated the increased ROS production induced by chilling stress (Fig. 6A-C). However, under low temperature condition, the $\mathrm{H}_{2} \mathrm{O}_{2}, \mathrm{O}_{2}^{-}$, and $\mathrm{OH}^{-}$levels increased markedly in the presence of PTIO or Flu compared with those under only chilling stress (Fig. 6A-C). In addition, the combination of SNP with Flu also markedly reduced the increased $\mathrm{H}_{2} \mathrm{O}_{2}, \mathrm{O}_{2}^{-}$, and $\mathrm{OH}^{-}$contents induced by chilling stress, whereas ABA in combination with PTIO significantly increased the contents of $\mathrm{H}_{2} \mathrm{O}_{2}, \mathrm{O}_{2}^{-}$, and $\mathrm{OH}^{-}$ compared with those of chilling treatment alone (Fig. 6A-C). There were no significant differences in $\mathrm{H}_{2} \mathrm{O}_{2}, \mathrm{O}_{2}^{-}$, or $\mathrm{OH}^{-}$ contents among all treatment groups at $25^{\circ} \mathrm{C}$ (Fig. 6A-C).

EfFects of ABA, SNP, PTIO, and Flu on antioxidant ENZYMES AND ANTIOXIDANT CONTENTS. The data presented in Fig. 7A-D indicated that the activities of SOD and GR increased significantly under conditions of chilling stress, whereas there were no changes in CAT and APX activities. Under conditions of chilling stress, the application of ABA, SNP, or SNP in combination with Flu induced marked increases in the activities of SOD, CAT, APX, and GR (Fig. 7A-D). However, the activities of SOD, APX, and GR decreased markedly in shoots treated with ABA in combination with PTIO, PTIO, or Flu compared with those under chilling stress alone (Fig. 7A-D). In addition, the activities of the above enzymes showed no obvious differences among all of the treatments at $25^{\circ} \mathrm{C}$.

As shown in Fig. 7E and F, chilling stress caused significant increases in AsA and GSH contents. The AsA and GSH contents increased by $60 \%$ and $75.4 \%$ in walnut shoots exposed 

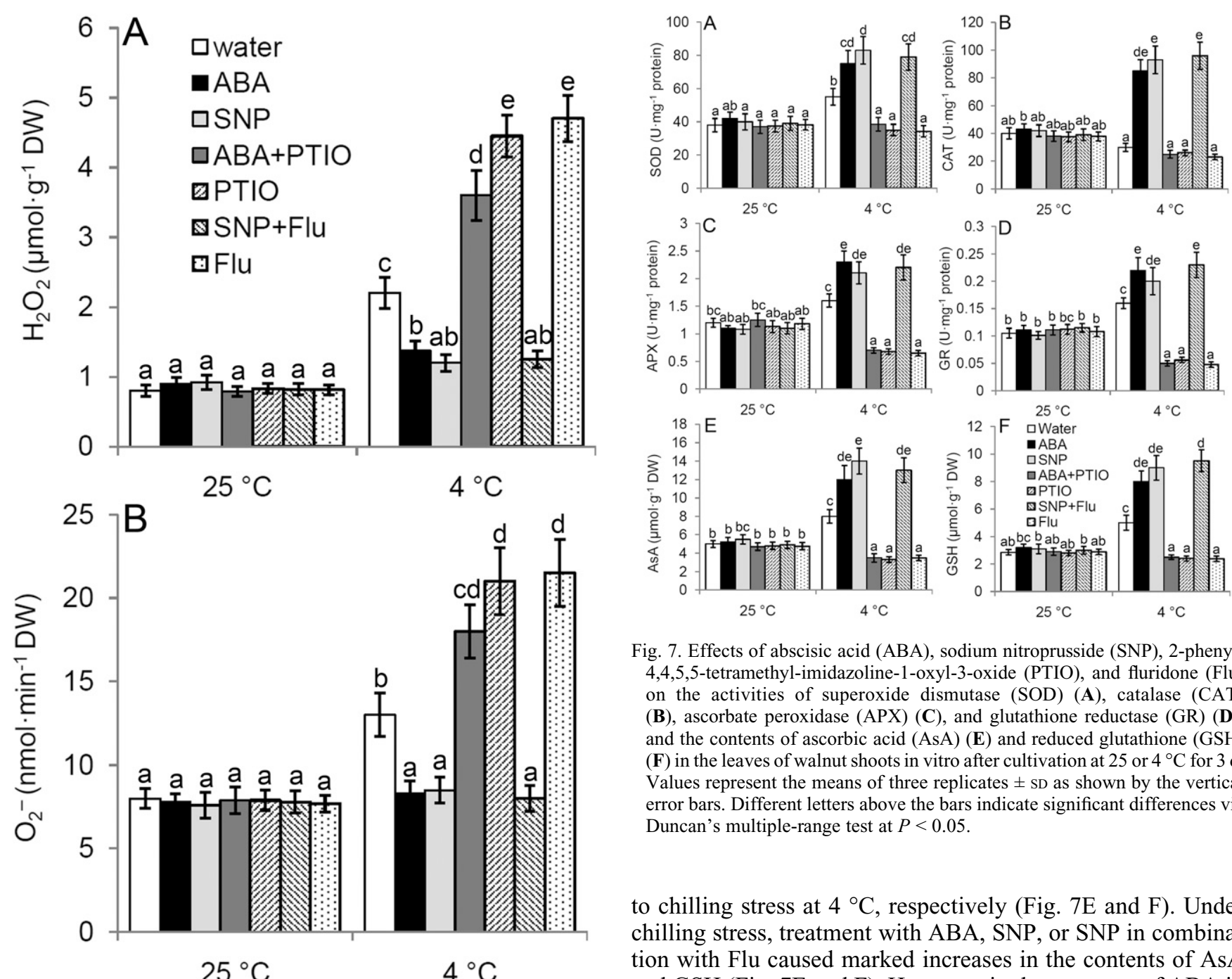

Fig. 7. Effects of abscisic acid (ABA), sodium nitroprusside (SNP), 2-phenyl4,4,5,5-tetramethyl-imidazoline-1-oxyl-3-oxide (PTIO), and fluridone (Flu) on the activities of superoxide dismutase (SOD) (A), catalase (CAT) (B), ascorbate peroxidase (APX) (C), and glutathione reductase (GR) (D), and the contents of ascorbic acid (AsA) (E) and reduced glutathione (GSH) (F) in the leaves of walnut shoots in vitro after cultivation at 25 or $4{ }^{\circ} \mathrm{C}$ for $3 \mathrm{~d}$. Values represent the means of three replicates \pm SD as shown by the vertical error bars. Different letters above the bars indicate significant differences via Duncan's multiple-range test at $P<0.05$.

to chilling stress at $4{ }^{\circ} \mathrm{C}$, respectively (Fig. 7E and F). Under chilling stress, treatment with ABA, SNP, or SNP in combination with Flu caused marked increases in the contents of AsA and GSH (Fig. 7E and F). However, in the presence of ABA in combination with PTIO, PTIO, or Flu, the contents of AsA and GSH decreased markedly compared with those exposed to chilling treatment alone (Fig. 7E and F). Besides, there were no significant changes in AsA or GSH contents among all treatments at $25^{\circ} \mathrm{C}$.

\section{Discussion}

Walnut, which has been cultivated for both its timber and edible nuts, is one of the most valuable and widely cultivated horticultural commodities in the world. Its nuts are a good source of flavonoids, sterols, polyphenols, and contains high amounts of essential dietary fatty acids, omega- 6 and omega- 3 (Ikhsana et al., 2016). However, walnut seedlings are susceptible to CI in northern China. Therefore, it is necessary to study the adaptive mechanism of walnut to chilling conditions. In the present study, walnut shoots in vitro were used to investigate the effects of $\mathrm{ABA}$ and $\mathrm{NO}$ on chilling resistance and activation of the antioxidant system under conditions of chilling stress. First, the results presented here provide theoretical basis and technical support for walnut chill-proof growing in real field conditions. Second, to reduce individual error walnut shoots in vitro that are obtained through vegetative propagation were used as experimental materials in this research. Vegetative propagation is used to produce progeny plants, which are 
genetically identical to a single source plant. Currently, vegetative propagation of walnut is achieved mainly by tissue culture, grafting or cutting rooting. However, the chilling tolerance of walnut was affected by different rootstocks in grafted seedlings (Li et al., 2017). And the cutting propagation difficulty of walnut is presented as poor rhizogenesis. Finally, this study also provides a substantial base for long-term storage of walnut cultures in vitro under low temperature condition.

Mechanisms underlying ABA-enhanced Chilling TOLERANCE IN WALNUT SHOOTS IN VITRO. ABA, a recognized plant stress hormone and growth inhibitor, has been shown to be an effective plant growth regulator of induction of cold acclimation (Theocharis et al., 2012). Application of exogenous ABA has been shown to induce chilling tolerance in various herbaceous plants and some trees (Guo et al., 2012; Meng et al., 2008). However, other studies indicated either little or no effect of ABA on the development of chilling tolerance (Gusta et al., 2005). These conflicting results suggest that there may be ABA-dependent and ABA-independent pathways associated with acclimation to cold stress (Roychoundhury et al., 2013; Theocharis et al., 2012). In the present study, exogenous ABA markedly alleviated electrolyte leakage and lipid peroxidation and significantly prevented the decreased $F_{\mathrm{v}} / F_{\mathrm{m}}$ and survival induced by chilling stress (Fig. 5A-D). These observations were consistent with those of other studies in Trigonobalanus doichangensis (Zheng et al., 2015), grapevine [Vitis vinifera (Karimi and Ershadi, 2015)], and Chorispora bungeana cells in suspension culture (Liu et al., 2011). ROS production is one of the earliest responses to abiotic stress in plants (Suzuki et al., 2012). The antioxidant defense system is rapidly induced in plant cells to combat the oxidant damage associated with ROS accumulation (Gill and Tuteja, 2010). Among the antioxidant enzyme systems, SOD is especially important because it catalyzes the removal of $\mathrm{O}_{2}^{-}$, the first $\mathrm{ROS}$ formed after exposure to chilling stress (Jaleel et al., 2009). Other antioxidant enzymes, including CAT, APX, and GR, convert $\mathrm{H}_{2} \mathrm{O}_{2}$ into water (Farnese et al., 2016; Lázaro et al., 2013). In combination with these enzymes, nonenzymatic antioxidants, such as AsA and GSH, also play crucial roles in scavenging ROS by acting as redox buffers in plant cells (Farnese et al., 2016). AsA and $\mathrm{GSH}$ can scavenge $\mathrm{O}_{2}^{-}$and $\mathrm{OH}^{-}$directly and reduce $\mathrm{H}_{2} \mathrm{O}_{2}$ into water (Ordoñez et al., 2014). Correspondingly, our results showed that, in the presence of ABA, the activities of SOD, CAT, APX, and GR and the contents of AsA and GSH were much higher than those seen with chilling treatment alone, whereas the contents of $\mathrm{H}_{2} \mathrm{O}_{2}, \mathrm{O}_{2}{ }^{-}$, and $\mathrm{OH}^{-}$were decreased (Figs. 6 and 7). These findings were consistent with previous studies, which showed that ABA enhanced antioxidant defenses and slowed down the accumulation of ROS caused by chilling stress (Liu et al., 2011; Wang et al., 2013). Therefore, these results suggest that exogenous ABA may confer chilling tolerance to walnut shoots.

To clarify the physiological roles of endogenous ABA in chilling stress responses, the ABA synthesis inhibitor Flu was used alone. Our results showed that application of Flu resulted in marked damage compared with those exposed to chilling stress alone, meaning that $F_{\mathrm{v}} / F_{\mathrm{m}}$ and survival declined markedly, electrolyte leakage and lipid peroxidation were significantly aggravated, ROS levels accumulated markedly, and the activities of SOD, APX, and GR and the contents of AsA and GSH decreased obviously (Figs. 5A-D, 6, and 7). Measurement of ABA content indicated that ABA level increased noticeably under chilling stress and Flu treatment markedly inhibited the chilling stress-induced increase in ABA (Fig. 5F). These data indicate that significant increase in ABA level under chilling stress is involved in chilling tolerance in walnut shoots.

ROLE OF NO IN THE CHILLING TOLERANCE OF WALNUT SHOOTS IN VITRO. Accumulating evidence suggests that NO plays important roles in the acclimation of plants to abiotic stress (Fancy et al., 2017). Application of exogenous NO to plants has been used to investigate the effects of NO in various physiological processes (Liu et al., 2010; Zhang et al., 2011). In the present study, application of SNP effectively prevented the increased electrolyte leakage, lipid peroxidation, and $F_{\mathrm{v}} / F_{\mathrm{m}}$ and survival suppression induced by chilling stress (Fig. 5A-D). These findings suggest that exogenous NO could protect walnut shoots from oxidative damage induced by chilling. NO may help plants resist oxidative injury via two pathways (Puyaubert and Baudouin, 2014). First, NO reacts directly with radical species such as $\mathrm{O}_{2}^{-}$and its intermediates in lipid peroxidation, acting as an antioxidant and blocking the lipid peroxidation process (Simontacchi et al., 2013). Second, NO could serve as a signaling molecule in the activation of cellular antioxidant defense systems (Fancy et al., 2017). As illustrated in a range of physiological contexts involving NO signaling, the posttranslational modification (PTM) of proteins via NO-based mechanisms plays important roles in NO signal transduction (Puyaubert and Baudouin, 2014). The major NO-dependent PTM is nitrosylation of cysteine residues (S-nitrosylation). Several antioxidant enzymes have been shown to be S-nitrosylated in plants under conditions of abiotic stress (Romero-Puertas et al., 2013). Some studies indicated that the enzymatic activities of SOD, CAT, APX, and GR are increased by chilling stress-induced Snitrosylation (Begara-Morales et al., 2013; Sehrawat and Deswal, 2014). In the present study, the activities of SOD, CAT, APX, and GR and the contents of AsA and GSH in the presence of SNP under chilling stress were much higher than those under chilling stress alone (Fig. 7). Application of SNP markedly reduced the accumulation of $\mathrm{H}_{2} \mathrm{O}_{2}, \mathrm{O}_{2}^{-}$, and $\mathrm{OH}^{-}$ induced by chilling (Fig. 6). Taken together, these observations indicate that $\mathrm{NO}$, as a bioactive antioxidant, can exert a protective effect against chilling-induced oxidative injury by scavenging ROS directly or by enhancing antioxidant defense systems.

Many recent studies have indicated increased production of NO in cold-stressed plants (Liu et al., 2010; Ziogas et al., 2013). However, the NO content decreased in bell pepper (Capsicum annuum) exposed to chilling conditions (Airaki et al., 2012). These studies indicated a certain degree of specificity in the role of endogenous NO under conditions of chilling stress, which may depend on the plant species or type of tissue. To clarify the physiological role of endogenous NO in chilling tolerance in walnut shoots in vitro, the NO scavenger PTIO was used. Our results showed that under chilling stress, treatment with PTIO resulted in significant oxidative damage in walnut shoots in comparison with that of chilling stress alone, which means that electrolyte leakage aggravated significantly, MDA and ROS levels accumulated remarkably, and $F_{\mathrm{v}} / F_{\mathrm{m}}$, survival, the activities of SOD, APX, and GR and the contents of AsA and GSH decreased obviously (Figs. 5-7). Detection of NO content indicated that NO level increased noticeably under chilling stress and PTIO treatment markedly inhibited the chilling stress-induced increase in NO (Fig. 5E). From these results, it 
is concluded that significant increase in NO level under chilling stress is involved in chilling tolerance in walnut shoots in vitro.

Possible relationship between ABA and NO IN WALNUT SHOOTS UNDER CHILLING CONDITIONS IN VITRO. There have been a number of investigations regarding the interaction between $\mathrm{NO}$ and ABA. In some cases, such as the induction of stomatal closure and upregulation of antioxidant enzyme gene transcription and activities, NO acts mainly as a downstream element in the ABA signaling pathway (Freschi, 2013; Zhang et al., 2007). On the other hand, ABA enhances oxidative stress tolerance by mediating ABA biosynthesis in maize leaves (Zhang et al., 2011). In addition, NO seems to counteract the effects of ABA in the regulation of seed dormancy (Sarath et al., 2007). These seemingly contradictory results may be due to the specificity of the NO-ABA interaction mechanisms, which may depend on physiological events or even the species of plant. These studies suggest that complex interactions may exist between ABA and $\mathrm{NO}$ in plants.

The present study indicated that prevention of NO accumulation by NO scavengers could eliminate the protective effect of exogenous ABA against CI (Fig. 5A-D). These results suggest that the protective effect of ABA may be mediated by NO. In addition, the increased NO content in ABA-treated walnut shoots under chilling conditions was suppressed by the NO scavenger PTIO or ABA synthesis inhibitor Flu (Fig. 5E), indicating that ABA triggered NO production under chilling stress. Moreover, ABA in combination with PTIO, PTIO, or Flu alone markedly blocked the activities of antioxidant enzymes, and the contents of AsA and GSH induced by ABA, while the $\mathrm{H}_{2} \mathrm{O}_{2}, \mathrm{O}_{2}^{-}$, and $\mathrm{OH}^{-}$contents were also enhanced under chilling stress (Figs. 6 and 7). Meanwhile, application of exogenous NO could also play a positive role in alleviating chilling damage and activation of antioxidant activities (Figs. 5A-D and 7). Conversely, SNP application induced the same ABA rise observed in control plants in response to chilling, and NO combined with Flu or Flu alone did not affect the SNP-induced protective effect against CI or activation of antioxidant activities in walnut shoots under chilling stress (Figs. 5A-D, F, and 7). Taken together, these findings indicate that ABA induced chilling tolerance and activation of antioxidant activities by promoting accumulation of NO, i.e., NO may act as a downstream element in ABA-induced chilling tolerance and activation of antioxidant activities under chilling stress.

In conclusion, the results of the present study indicated that both $\mathrm{ABA}$ and $\mathrm{NO}$ enhanced chilling tolerance and activated the antioxidant defense system in walnut shoots under chilling stress in vitro. To our knowledge, this is the first report that ABA-triggered production of NO may play a crucial role in ABA-induced chilling tolerance by activating antioxidant defense systems and thus suppressing the accumulation of ROS in walnut shoots under low temperature conditions in vitro. NO may be a component of the ABA-induced plant acclimation response to chilling stress. However, further evidence is required to determine the complex molecular network operating under conditions of chilling stress mediated by ABA and NO. Further studies are needed to elucidate how ABA regulates NO generation under conditions of chilling stress.

\section{Literature Cited}

Airaki, M., M. Leterrier, R.M. Mateos, R. Valderrama, M. Chaki, J.B. Barroso, L.A. Del Río, J.M. Palma, and F.J. Corpas. 2012. Metabolism of reactive oxygen species and reactive nitrogen species in pepper (Capsicum annuum L.) plants under low temperature stress. Plant Cell Environ. 35:281-295.

Asgher, M., T.S. Per, A. Masood, M. Fatma, L. Freschi, F.J. Corpas, and N.A. Khan. 2017. Nitric oxide signaling and its crosstalk with other plant growth regulators in plant responses to abiotic stress. Environ. Sci. Pollut. Res. 24:2273-2285.

Begara-Morales, J.C., B. Sánchez-Calvo, M. Chaki, R. Valderrama, C. Mata-Pérez, J. López-Jaramillo, M.N. Padilla, A. Carreras, F.J. Corpas, and J.B. Barroso. 2013. Dual regulation of cytosolic ascorbate peroxidase (APX) by tyrosine nitration and S-nitrosylation. J. Expt. Bot. 65:527-538.

Bradford, M.M. 1976. A rapid and sensitive method for the quantification of microgram quantities of protein utilizing the principle of protein-dye binding. Anal. Biochem. 72:248-254.

Dong, C.J., L. Li, Q.M. Shang, X.Y. Liu, and Z.G. Zhang. 2014. Endogenous salicylic acid accumulation is required for chilling tolerance in cucumber (Cucumis sativus L.) seedlings. Planta 240:687-700.

Fancy, N.N., A.K. Bahlmann, and G.J. Loake. 2017. Nitric oxide function in plant abiotic stress. Plant Cell Environ. 40:462-472.

Farnese, F.S., P.E. Menezes-Silva, G.S. Gusman, and J.A. Oliveira. 2016. When bad guys become good ones: The key role of reactive oxygen species and nitric oxide in the plant responses to abiotic stress. Front. Plant Sci. 7:471.

Freschi, L. 2013. Nitric oxide and phytohormone interactions: Current status and perspectives. Front. Plant Sci. 4:398.

Gill, S.S. and N. Tuteja. 2010. Reactive oxygen species and antioxidant machinery in abiotic stress tolerance in crop plants. Plant Physiol. Biochem. 48:909-930.

Gou, J.Q., S.H. Strauss, C.J. Tsai, K. Fang, Y.R. Chen, X.N. Jiang, and V.B. Busov. 2010. Gibberellins regulate lateral root formation in Populus through interactions with auxin and other hormones. Plant Cell 22:623-639.

Griffith, O.W. 1980. Determination of glutathione and glutathione disulfide using glutathione reductase and 2-vinylpyridine. Anal. Biochem. 106:207-212.

Guo, W.L., R.G. Chen, Z.H. Gong, Y.X. Yin, S.S. Ahmed, and Y.M. He. 2012. Exogenous abscisic acid increases antioxidant enzymes and related gene expression in pepper (Capsicum annuum) leaves subjected to chilling stress. Genet. Mol. Res. 11:4063-4080.

Guo, Z., W. Ou, S. Lu, and Q. Zhong. 2006. Differential responses of antioxidative system to chilling and drought in four rice cultivars differing in sensitivity. Plant Physiol. Biochem. 44:828-836.

Gusta, L.V., R. Trischuk, and C.J. Weiser. 2005. Plant cold acclimation: The role of abscisic acid. J. Plant Growth Regul. 24:308-318.

Hu, Z.R., E. Amombo, M.M. Gitau, A.Y. Bi, H.H. Zhu, L. Zhang, L. Chen, and J.M. Fu. 2017. Changes of antioxidant defense system and fatty acid composition in bermudagrass under Chilling Stress. J. Amer. Soc. Hort. Sci. 142:101-109.

Ikhsana, A.S., H. Topçua, M. Sütyemezb, and S. Kafkas. 2016. Novel 307 polymorphic SSR markers from BAC-end sequences in walnut (Juglans regia L.): Effects of motif types and repeat lengths on polymorphism and genetic diversity. Sci. Hort. 213:1-4.

Jaleel, C.A., K. Riadh, R. Gopi, P. Manivannan, J. Inès, H.J. Al-Juburi, C.X. Zhao, H.B. Shao, and R. Panneerselvam. 2009. Antioxidant defense response: Physiological plasticity in higher plants under abiotic constraints. Acta Physiol. Plant. 31:427-436.

Karimi, R. and A. Ershadi. 2015. Role of exogenous abscisic acid in adapting of 'Sultana' grapevine to low-temperature stress. Acta Physiol. Plant. 37:151-161.

Law, M.Y., S.A. Charles, and B. Halliwell. 1983. Glutathione and ascorbic acid in spinach (Spinacia oleracea) chloroplasts. Biochem. J. 210:899-903.

Lázaro, J.J., A. Jiménez, D. Camejo, I. Iglesias-Baena, M.C. Martí, A. Lázaro-Payo, S. Barranco-Medina, and F. Sevilla. 2013. Dissecting the integrative antioxidant and redox systems in plant mitochondria. Effect of stress and S-nitrosylation. Front. Plant Sci. 4:460. 
Li, H., M.M. Liang, S. Zhao, B.G. Li, H. Li, and G.H. Qi. 2017. Effects of different rootstocks on cold resistance of 'Lyuling' walnut. Northern Hort. 3:25-31 (in Chinese).

Liu, Y.J., H.F. Jiang, Z.G. Zhao, and L.Z. An. 2010. Nitric oxide synthase like activity-dependent nitric oxide production protects against chilling-induced oxidative damage in Chorispora bungeana suspension cultured cells. Plant Physiol. Biochem. 48:936-944.

Liu, Y.J., H.F. Jiang, Z.G. Zhao, and L.Z. An. 2011. Abscisic acid is involved in brassinosteroids-induced chilling tolerance in the suspension cultured cells from Chorispora bungeana. J. Plant Physiol. 168:853-862.

Manai, J., J. Kalai, H. Gouia, and F.J. Corpas. 2014. Exogenous nitric oxide (NO) ameliorates salinity-induced oxidative stress in tomato (Solanum lycopersicum) plants. J. Soil Sci. Plant Nutr. 14:433-446. Meng, F.Z., L.P. Hu, S.H. Wang, X.L. Sui, L. Wei, Y.X. Wei, J.L. Sun, and Z.X. Zhang. 2008. Effects of exogenous abscisic acid (ABA) on cucumber seedling leaf carbohydrate metabolism under low temperature. Plant Growth Regulat. 56:233-244.

Neill, S., J. Bright, R. Desikan, J. Hancock, J. Harrison, and I. Wilson. 2008. Nitric oxide evolution and perception. J. Expt. Bot. 59:25-35.

Ordoñez, N.M., C. Marondedze, L. Thomas, S. Pasqualini, L. Shabala, S. Shabala, and C. Gehring. 2014. Cyclic mononucleotides modulate potassium and calcium flux responses to $\mathrm{H}_{2} \mathrm{O}_{2}$ in Arabidopsis roots. FEBS Lett. 588:1008-1015.

Pei, D., L.C. Yuan, Q.M. Wang, and R.S. Gu. 2007. Factors affecting rooting of in vitro shoots of walnut cultivars. J. Hort. Sci. Biotechnol. 82:223-226.

Puyaubert, J. and E. Baudouin. 2014. New clues for a cold case: Nitric oxide response to low temperature. Plant Cell Environ. 37:26232630.

Romero-Puertas, M.C., M. Rodríguez-Serrano, and L.M. Sandalio. 2013. Protein S-nitrosylation in plants under abiotic stress: An overview. Front. Plant Sci. 4:373.

Roychoundhury, A., S. Paul, and S. Basu. 2013. Cross-talk between abscisic acid-dependent and abscisic acid-independent pathways during abiotic stress. Plant Cell Rpt. 32:985-1006.

Sarath, G., G. Hou, L.M. Baird, and R.B. Mitchell. 2007. Reactive oxygen species, ABA and nitric oxide interactions on the germination of warm-season $\mathrm{C}_{4}$-grasses. Planta 226:697-708.

Simontacchi, M., C. García-Mata, C.G. Bartoli, G.E. Santa-María, and L. Lamattina. 2013. Nitric oxide as a key component in hormoneregulated processes. Plant Cell Rpt. 32:853-866.

Sehrawat, A. and R. Deswal. 2014. S-Nitrosylation analysis in Brassica juncea apoplast highlights the importance of nitric oxide in cold-stress signaling. J. Proteome Res. 13:2599-2619.

Soloklui, A.A.G., A. Ershadi, and E. Fallahi. 2012. Evaluation of cold hardiness in seven Iranian commercial pomegranate (Punica granatum L.) cultivars. HortScience 47:1821-1825.

Suzuki, N., S. Koussevitzky, R. Mittler, and G. Miller. 2012. ROS and redox signaling in the response of plants to abiotic stress. Plant Cell Environ. 35:259-270.
Theocharis, A., C. Clément, and E.A. Barka. 2012. Physiological and molecular changes in plants grown at low temperatures. Planta 235:1091-1105.

Venzhik, Y., V. Talanova, and A. Titov. 2016. The effect of abscisic acid on cold tolerance and chloroplasts ultrastructure in wheat under optimal and cold stress conditions. Acta Physiol. Plant. 38:63-72.

Wang, G.L., W. Miao, J.Y. Wang, D.R. Ma, J.Q. Li, and W.F. Chen. 2013. Effect of exogenous abscisic acid on antioxidant system in weedy and cultivated rice with different chilling sensitivity under chilling stress. J. Agron. Crop Sci. 199:200-208.

Wang, L.Z., L.M. Wang, H.T. Xiang, Y. Luo, R. Li, Z.J. Li, C.Y. Wang, and Y. Meng. 2016. Relationship of photosynthetic efficiency and seed-setting rate in two contrasting rice cultivars under chilling stress. Photosynthetica 54:581-588.

Zhang, A.Y., M.Y. Jiang, J.H. Zhang, H.D. Ding, S.C. Xu, X.L. Hu, and M.P. Tan. 2007. Nitric oxide induced by hydrogen peroxide mediates abscisic acid-induced activation of the mitogen-activated protein kinase cascade involved in antioxidant defense in maize leaves. New Phytol. 175:36-50.

Zhang, A.Y., J. Zhang, J.H. Zhang, N.H. Ye, H. Zhang, M.P. Tan, and M.Y. Jiang. 2011. Nitric oxide mdiates brassinosteroid-induced ABA biosynthesis involved in oxidative stress tolerance in maize leaves. Plant Cell Physiol. 52:181-192.

Zhang, J.X., X.C. Wu, R.X. Niu, Y. Liu, N. Liu, W.R. Xu, and Y.J. Wang. 2012. Cold-resistence evaluation in 25 wild grape species. Vitis 51:153-160.

Zhang, Y.M., J.L. Tan, Z.F. Guo, S.Y. Lu, S.J. He, W. Shu, and B.Y. Zhou. 2009. Increased abscisic acid levels in transgenic tobacco over-expressing 9 cis-epoxycarotenoid dioxygenase influence $\mathrm{H}_{2} \mathrm{O}_{2}$ and NO production and antioxidant defences. Plant Cell Environ. 32:509-519.

Zhao, L., J.X. He, X.M. Wang, and L.X. Zhang. 2008. Nitric oxide protects against polyethylene glycol-induced oxidative damage in two ecotypes of reed suspension cultures. J. Plant Physiol. 165:182-191.

Zheng, Y.L., W.Q. Li, and W.B. Sunday. 2015. Effects of acclimation and pretreatment with abscisic acid or salicylic acid on tolerance of Trigonobalanus doichangensis to extreme temperatures. Biol. Plant. 59:382-388.

Zhou, B.Y. and Z.F. Guo. 2009. Calcium is involved in the abscisic acid-induced ascorbate peroxidase, superoxide dismutase and chilling resistance in Stylosanthes guianensis. Biol. Plant. 53:63-68.

Zhou, B.Y., Z.F. Guo, J.P. Xing, and B.R. Huang. 2005. Nitric oxide is involved in abscisic acid-induced antioxidant activities in Stylosanthes guianensis. J. Expt. Bot. 56:3223-3228.

Zhou, B.Y., J.H. Wang, Z.F. Guo, H.Q. Tan, and X.C. Zhu. 2006. A simple colorimetric method for determination of hydrogen peroxide in plant tissues. Plant Growth Regulat. 49:113-118.

Ziogas, V., G. Tanou, P. Filippou, G. Diamantidis, M. Vasilakakis, V. Fotopoulos, and A. Molassiotis. 2013. Nitrosative responses in citrus plants exposed to six abiotic stress conditions. Plant Physiol. Biochem. 68:118-126. 